\title{
Nucleon properties from basis light front quantization
}

\author{
Chandan Mondal ${ }^{* 1}$, Siqi Xu ${ }^{1,2}$, Jiangshan Lan ${ }^{1,2,3}$, Xingbo Zhao ${ }^{1,2}$, Yang $\mathbf{L i}^{4,5,6}$, Henry \\ Lamm $^{7}$, James P. Vary ${ }^{4}$ \\ ${ }^{1}$ Institute of Modern Physics, Chinese Academy of Sciences, Lanzhou 730000, China \\ ${ }^{2}$ School of Nuclear Science and Technology, University of Chinese Academy of Sciences, \\ Beijing 100049, China \\ ${ }^{3}$ Lanzhou University, Lanzhou 730000, China \\ ${ }^{4}$ Department of Physics and Astronomy, Iowa State University, Ames, IA 50011, USA \\ ${ }^{5}$ Hebei Key Laboratory of Compact Fusion, Langfang 065001, China \\ ${ }^{6}$ ENN Science and Technology Development Co., Ltd., Langfang 065001, China \\ ${ }^{7}$ Department of Physics, University of Maryland, College Park, Maryland 20742, USA
}

\begin{abstract}
We investigate the electromagnetic properties of the nucleon using the framework of basis light front quantization. We consider the light front wave functions obtained by diagonalizing an effective Hamiltonian consisting of the holographic quantum chromodynamics confinement potential, a longitudinal confinement and a one-gluon exchange interaction with fixed coupling. The electromagnetic radii of the nucleon are also evaluated.
\end{abstract}

XXVII International Workshop on Deep-Inelastic Scattering and Related Subjects - DIS2019 8-12 April, 2019

Torino, Italy

${ }^{*}$ Speaker. 


\section{Introduction}

Basis light front quantization (BLFQ) is a nonperturbative approach which has been developed for solving many-body bound-state problems in quantum field theories [1, 2, 3, 4]. It is a Hamiltonian formalism incorporating the advantages of the light front dynamics [5, 6]. This formalism has been successfully applied to QED systems including the electron anomalous magnetic moment [4] and the strong coupling bound-state positronium problem [2]. It has also been applied to heavy quarkonia [3, 7] and $B_{c}$ mesons [8] both as QCD bound states. Recently, the BLFQ approach using a Hamiltonian that includes the color singlet Nambu-Jona-Lasinio interaction to account for the chiral dynamics has been applied to the light mesons $[9,10,11]$. In this work, we apply the BLFQ formalism to the nucleon and study the electromagnetic form factors using the light front wave functions (LFWFs) obtained by diagonalizing the effective light front Hamiltonian in the constituent valence quark representation. In the effective Hamiltonian, we adopt interactions including the light front holographic QCD in the transverse direction [12], a longitudinal confinement [3], and a one-gluon exchange interaction with a fixed coupling in the framework of BLFQ.

In the light front formalism for a spin 1/2 composite system the Dirac and Pauli form factors $F_{1}\left(q^{2}\right)$ and $F_{2}\left(q^{2}\right)$ are identified with the helicity-conserving and helicity-flip matrix elements of the vector current. These form factors can be expressed in terms of overlap integrals as [13]

$$
\begin{aligned}
F_{1}^{q}\left(q^{2}\right)= & \sum_{n, \lambda_{i}} \int \prod_{i=1}^{n} \frac{d x_{i} d^{2} k_{\perp i}}{16 \pi^{3}} 16 \pi^{3} \delta\left(1-\sum_{j} x_{j}\right) \delta^{2}\left(\sum_{j=1}^{n} k_{\perp j}\right) \\
& \times \psi_{n}^{\uparrow *}\left(x_{i}^{\prime}, k_{\perp i}^{\prime}, \lambda_{i}\right) \psi_{n}^{\uparrow}\left(x_{i}, k_{\perp i}, \lambda_{i}\right), \\
\frac{-\left(q^{1}-i q^{2}\right)}{2 M} F_{2}^{q}\left(q^{2}\right)= & \sum_{n, \lambda_{i}} \int \prod_{i=1}^{n} \frac{d x_{i} d^{2} k_{\perp i}}{16 \pi^{3}} 16 \pi^{3} \delta\left(1-\sum_{j} x_{j}\right) \delta^{2}\left(\sum_{j=1}^{n} k_{\perp j}\right) \\
& \times \psi_{n}^{\uparrow *}\left(x_{i}^{\prime}, k_{\perp i}^{\prime}, \lambda_{i}\right) \psi_{n}^{\downarrow}\left(x_{i}, k_{\perp i}, \lambda_{i}\right),
\end{aligned}
$$

where $\psi_{n}^{\Lambda}\left(x_{i}, k_{\perp i}, \lambda_{i}\right)$ is the boost invariant light-front wave functions. $\lambda_{i}$ and $\Lambda$ are the lightcone helicities of the parton and the composite system, respectively. For the struck parton $x^{\prime}{ }_{1}=$ $x_{1} ; k_{\perp 1}^{\prime}=k_{\perp 1}+\left(1-x_{1}\right) q_{\perp}$ and $x_{i}^{\prime}=x_{i} ; k_{\perp i}^{\prime}=k_{\perp i}-x_{i} q_{\perp}$ for the spectators $(i=2, \ldots . n)$. We consider the frame where the momentum transfer $q=\left(0,0, \mathbf{q}_{\perp}\right)$, thus $Q^{2}=-q^{2}=\mathbf{q}_{\perp}^{2}$.

Under charge and isospin symmetry, it is straightforward to write down the flavor decomposition of the nucleon form factors $[14,15]$

$$
F_{i}^{p(n)}=e_{u} F_{i}^{u(d)}+e_{d} F_{i}^{d(u)} \quad(i=1,2)
$$

with the normalizations $F_{1}^{u}(0)=2, F_{2}^{u}(0)=\kappa_{u}$ and $F_{1}^{d}(0)=1, F_{2}^{d}(0)=\kappa_{d}$ where the anomalous magnetic moments for the up and the down quarks are $\kappa_{u}=2 \kappa_{p}+\kappa_{n}=1.673$ and $\kappa_{d}=\kappa_{p}+2 \kappa_{n}=$ -2.033 . The nucleon Sachs form factors are written in terms of Dirac and Pauli form factors as

$$
G_{E}^{N}\left(Q^{2}\right)=F_{1}^{N}\left(Q^{2}\right)-\frac{Q^{2}}{4 M^{2}} F_{2}^{N}\left(Q^{2}\right), \quad G_{M}^{N}\left(Q^{2}\right)=F_{1}^{N}\left(Q^{2}\right)+F_{2}^{N}\left(Q^{2}\right),
$$

and the electromagnetic radii are defined by

$$
\left\langle r_{E}^{2}\right\rangle^{N}=-\left.6 \frac{d G_{E}^{N}\left(Q^{2}\right)}{d Q^{2}}\right|_{Q^{2}=0}, \quad\left\langle r_{M}^{2}\right\rangle^{N}=-\left.\frac{6}{G_{M}^{N}(0)} \frac{d G_{M}^{N}\left(Q^{2}\right)}{d Q^{2}}\right|_{Q^{2}=0} .
$$




\section{Effective light front Hamiltonian}

The structures of the bound states are encoded in the LFWFs which are the eigenfunctions of the light front eigenvalue problem: $H_{\text {eff }}|\Psi\rangle=M^{2}|\Psi\rangle$, where $H_{\text {eff }}$ is the effective Hamiltonian of the system with the mass squared $M^{2}$ eigenvalue. In the valence Fock sector, the effective Hamiltonian for the nucleons that we adopt is given by

$$
\begin{aligned}
H_{\mathrm{eff}}= & \sum_{a} \frac{\vec{k}_{a \perp}^{2}+m_{a}^{2}}{x_{a}}+\frac{1}{2} \sum_{a, b}\left[\kappa_{T}^{4} x_{a} x_{b}\left(\vec{r}_{a \perp}-\vec{r}_{b \perp}\right)^{2}-\frac{\kappa_{L}^{4}}{\left(m_{a}+m_{b}\right)^{2}} \partial_{x_{a}}\left(x_{a} x_{b} \partial_{x_{b}}\right)\right] \\
& +\frac{1}{2} \sum_{a, b} \frac{C_{F} 4 \pi \alpha_{s}\left(Q_{a b}^{2}\right)}{Q_{a b}^{2}} \bar{u}_{s_{a}^{\prime}}\left(k_{a}^{\prime}\right) \gamma^{\mu} u_{s_{a}}\left(k_{a}\right) \bar{u}_{s_{b}^{\prime}}\left(k_{b}^{\prime}\right) \gamma^{v} u_{s_{b}}\left(k_{b}\right) g_{\mu \nu},
\end{aligned}
$$

where $\sum_{a} x_{a}=1$, and $\sum_{a} \mathbf{k}_{a \perp}=0 . m_{a / b}$ is the mass of the specified quark, and $\kappa_{L}\left(\kappa_{T}\right)$ is the strength of the longitudinal (transverse) confinement. $\vec{\zeta}_{\perp} \equiv \sqrt{x_{a} x_{b}} \vec{r}_{\perp}$ is the holographic variable [12], where $\vec{r}_{\perp}=\vec{r}_{a \perp}-\vec{r}_{b \perp}$ is the transverse separation between two quarks, $\partial_{x} f\left(x, \vec{\zeta}_{\perp}\right)=\partial f\left(x, \vec{\zeta}_{\perp}\right) /\left.\partial x\right|_{\vec{\zeta}}$. $Q_{a b}^{2}=-q^{2}=-(1 / 2)\left(k_{a}^{\prime}-k_{a}\right)^{2}-(1 / 2)\left(k_{b}^{\prime}-k_{b}\right)^{2}$ is the average momentum transfer squared, $C_{F}=$ $-2 / 3$ is the color factor. $g_{\mu \nu}$ is the metric tensor and $\alpha_{s}$ is the running coupling which we replace by a constant for simplicity. Note that we use different quark masses in the kinetic energy from those in the other terms of the effective light front Hamiltonian to attempt to account for the effects of higher Fock components and the other QCD interactions.

The light front mass eigenvalue problem is solved in the BLFQ approach using a single-particle representation $[1,2,4]$. In this approach, we choose the two dimensional harmonic oscillator ('2D HO') basis in the transverse direction and the discretized plane-wave basis in the longitudinal direction. Each single-particle basis state is identified using four quantum numbers, $\bar{\alpha}=\{k, n, m, \lambda\}$. The longitudinal momentum of the particle is characterized by the first quantum number $k$. In the longitudinal direction $x^{-}$, we constrain the system to a box of length $2 L$, and impose (anti-) periodic boundary conditions on (fermions) bosons ${ }^{1}$. As a result, the longitudinal momentum $p^{+}=2 \pi k / L$ is discretized, where the dimensionless quantity $k=1,2,3, \ldots$ for bosons and $k=1 / 2,3 / 2,5 / 2, \ldots$ for fermions. The zero mode for bosons is neglected. In the many-body basis, all basis states are selected to have the same total longitudinal momentum $P^{+}=\sum_{i} p_{i}^{+}$, where the sum is over the particles in a particular basis state. One then parameterizes $P^{+}$using a dimensionless variable $K_{\max }=\sum_{i} k_{i}$ such that $P^{+}=2 \pi K_{\max } / L$. For a given particle $i$, the longitudinal momentum fraction $x$ is defined as $x_{i}=p_{i}^{+} / P^{+}=k_{i} / K_{\max }$. The next two quantum numbers, $n$ and $m$, denote radial excitation and angular momentum projection, respectively, of the particle within the 2D HO basis in the transverse direction. The choice of the $2 \mathrm{D}$ HO basis for BLFQ is made because the HO potential is our confining potential, and therefore its wave functions should form a reasonable basis for the expansion of the long-distance behavior of the solutions. In terms of the dimensionless transverse momentum variable $\vec{v}_{\perp}\left(=\vec{k}_{\perp} / b\right)$, the ortho-normalized $2 \mathrm{D}$ HO basis function reads

$$
\phi_{n m}\left(\vec{v}_{\perp}\right)=\sqrt{\frac{n !}{(n+|m|) ! \pi}} e^{i m \theta} v^{|m|} e^{-v^{2} / 2} L_{n}^{|m|}\left(v^{2}\right),
$$

\footnotetext{
${ }^{1}$ Although we do not include dynamical gluons in the present work, we specify their corresponding modes as seen for dynamical photon in Ref. [4], for example.
} 
where $v=\left|\vec{v}_{\perp}\right|, \theta=\arg \vec{v}_{\perp}, L_{n}^{\alpha}(x)$ is the associated Laguerre polynomial, and $b$ is the HO basis scale with dimension of mass. For the spin degrees of freedom, the quantum number $\lambda$ is used to label the helicity of the particle.

In order to numerically diagonalize $H_{\text {eff }}$, the infinite dimensional basis must be truncated. In BLFQ, two levels of truncation scheme are implemented. First, the number of Fock sectors in the basis is restricted. For example, the nucleon state can be expressed schematically as

$$
|N\rangle_{\text {phys }}=a|q q q\rangle+b|q q q g\rangle+c|q q q q \bar{q}\rangle+\cdots .
$$

In this work, we limit ourselves to only the leading Fock sector $|q q q\rangle$. Thus, the nucleon basis state can be written as

$$
\left|N_{\text {phys }}^{S_{z}}\right\rangle=\left|k_{q_{1}}, n_{q_{1}}, m_{q_{1}}, \lambda_{q_{1}}\right\rangle \otimes\left|k_{q_{2}}, n_{q_{2}}, m_{q_{2}}, \lambda_{q_{2}}\right\rangle \otimes\left|k_{q_{3}}, n_{q_{3}}, m_{q_{3}}, \lambda_{q_{3}}\right\rangle .
$$

Second, within each Fock-sector, further truncation is still needed to reduce the basis to a finite dimension. We introduce a truncation parameter $K_{\max }$ on the longitudinal direction such that, $\sum_{i} k_{i}=K_{\max }$. In the transverse direction, we require the total transverse quantum number $N_{\alpha}=\sum_{l}\left(2 n_{l}+\left|m_{l}\right|+1\right)$ for multi-particle basis state $|\alpha\rangle$ satisfying $N_{\alpha} \leq N_{\max }$, where $N_{\max }$ is a chosen truncation parameter. The continuum limit corresponds to both $K_{\max }, N_{\max } \rightarrow \infty$. In addition, our many body states have well defined values of the total angular momentum projection $M_{J}=\sum_{i}\left(m_{i}+\lambda_{i}\right)$, where $\lambda$ is the fourth quantum number which corresponds the helicity of the particle.

We obtain the spectrum and the light front wave functions numerically by diagonalizing the effective Hamiltonian given in Eq.(2.1) with the basis representation given by Eq. (2.4). Using the resulting light front wave functions $\psi_{n}$, we evaluate the electromagnetic form factors of the nucleon. The parameters are tuned by hand to provide a reasonable fit to the electromagnetic properties of the nucleons.

\section{Numerical results}

In Fig. 1(a) and 1(b), we show the electric and magnetic Sach's form factors for the proton whereas the same form factors for the neutron are shown in Fig. 1(c) and 1(d). We set the confining strength, $\kappa_{L}=\kappa_{T}=0.4 \mathrm{GeV}$ in both the longitudinal and transverse confinements. The bands represent the range of our results due to increasing the basis from $N_{\max }=6$ to $N_{\max }=8$ both with $K_{\max }=10$. The couplings corresponding to $N_{\max }=6$ and $N_{\max }=8$ are $\alpha_{s}=1.7$ and $\alpha_{s}=1.4$, respectively. In order to attempt to simulate the effect of higher Fock component and the other QCD interactions, we use masses for up and down quarks in the kinetic energy i.e. $m_{\mathrm{u} / \mathrm{KE}}=0.6$ $\mathrm{GeV}$ and $m_{\mathrm{d} / \mathrm{KE}}=0.57 \mathrm{GeV}$ and a different quark mass in the one gluon exchange interaction and in longitudinal confinement and in longitudinal confining term $m_{\mathrm{q} / \mathrm{OGE}}=0.3 \mathrm{GeV}$. Overall we obtain a reasonable agreement between theory and experiment for the nucleon electric form factors. At low $Q^{2}$, the magnetic form factor for the proton is also in reasonable agreement with the data. However, the theoretical magnetic form factor for the neutron exhibits more significant deviation from the data which indicates there is room to further improve the BLFQ results. It should also be noted that these results are obtained from a finite basis and thus, there may be finite basis effect that should be further examined in future research. 
(a)

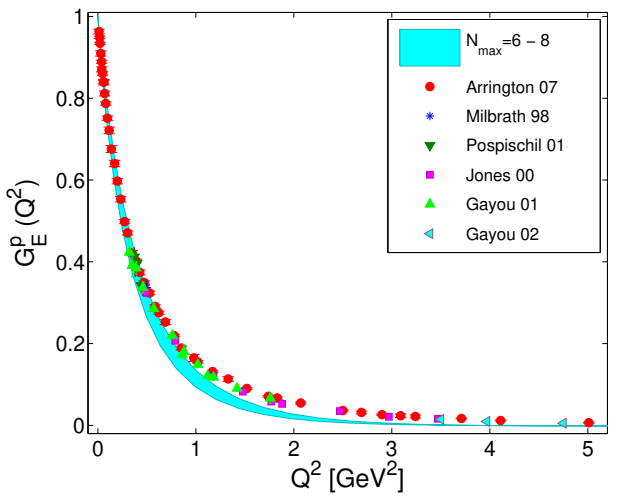

(c)

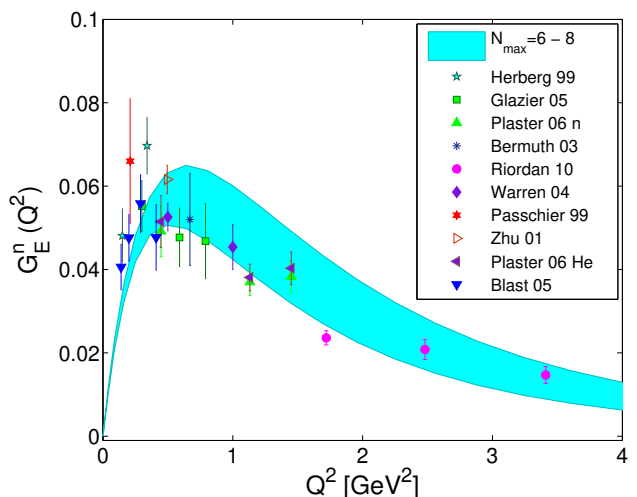

(b)

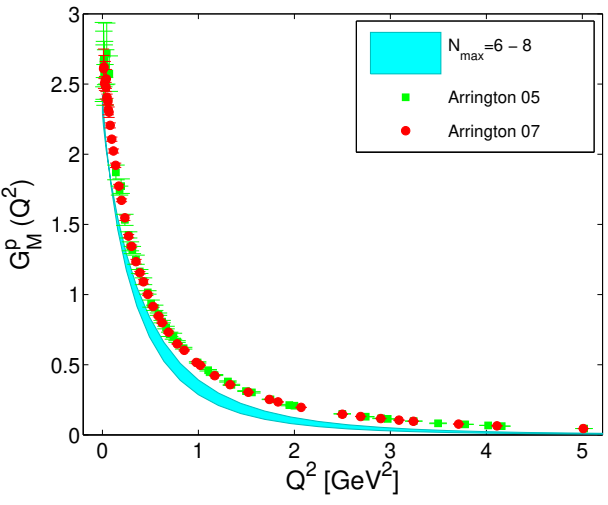

(d)

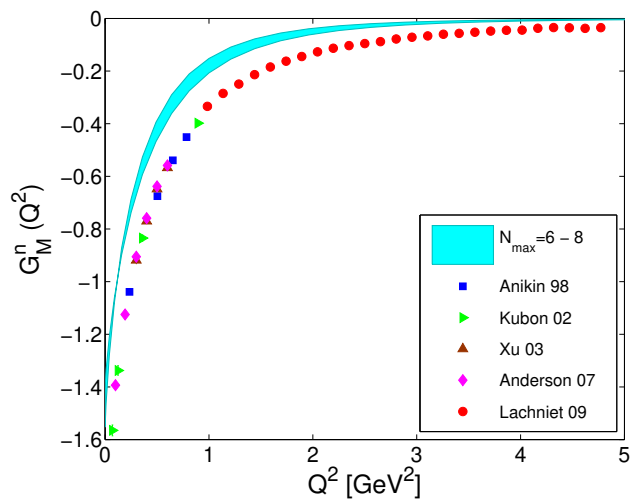

Figure 1: (Color online) BLFQ results for the nucleon Sach's form factors; (a) $G_{E}^{p}\left(Q^{2}\right)$, (b) $G_{M}^{p}\left(Q^{2}\right)$ for the proton and (c) $G_{E}^{n}\left(Q^{2}\right)$, (d) $G_{M}^{n}\left(Q^{2}\right)$ for the neutron. We use the confining strength, $\kappa_{L}=\kappa_{T}=0.4 \mathrm{GeV}$. The quark masses in the kinetic energy are $m_{\mathrm{u} / \mathrm{KE}}=0.6 \mathrm{GeV}$ and $m_{\mathrm{d} / \mathrm{KE}}=0.57 \mathrm{GeV}$, whereas the quark mass in one gluon exchange interaction $m_{\mathrm{q} / \mathrm{OGE}}=0.3 \mathrm{GeV}$. The bands correspond the range for $\left[N_{\max }=6, \alpha_{s}=1.7\right]$ and [ $N_{\max }=8, \alpha_{s}=1.4$ ] both with $K_{\max }=10$. We choose the value of HO parameter $b$ to be the same as $\kappa_{L}\left(\kappa_{T}\right)$ i.e. $b=0.4 \mathrm{GeV} . m_{g}(=0.05 \mathrm{GeV})$ is a small gluon mass regulator used for numerical convenience. The experimental data can be found in Ref. [14, 15].

From the Sachs form factors we also compute the electromagnetic radii of the nucleons. We quote the radii in Table 1, the experimental values are taken from the Ref. [16]. Here again, we find reasonable agreement with experiment for the charge radii. However the BLFQ results for magnetic radii are overestimated compared to the experimental data.

\section{Conclusions}

The electromagnetic form factors for the nucleon have been presented using the BLFQ approach. The form factors have been evaluated from the overlaps of the light front wave functions which were obtained by diagonalizing the effective Hamiltonian. In our model, we consider the holographic QCD confinement potential, a longitudinal confinement, and a one-gluon exchange interaction with fixed coupling in the effective light front Hamiltonian. We observed a reasonable agreement of our results for the nucleon electric form factors with the experimental data. On the 


\begin{tabular}{|c|c|c|}
\hline Quantity & BLFQ & Measured data [16] \\
\hline$r_{E}^{p}$ & $0.887 \mathrm{fm}$ & $0.877 \pm 0.005 \mathrm{fm}$ \\
$r_{M}^{p}$ & $1.008 \mathrm{fm}$ & $0.777 \pm 0.016 \mathrm{fm}$ \\
\hline$\left\langle r_{E}^{2}\right\rangle^{n}$ & $-0.1398 \mathrm{fm}^{2}$ & $-0.1161 \pm 0.0022 \mathrm{fm}^{2}$ \\
$r_{M}^{n}$ & $1.204 \mathrm{fm}$ & $0.862_{-0.008}^{+0.009} \mathrm{fm}$ \\
\hline
\end{tabular}

Table 1: Electromagnetic radii of the nucleons. The BLFQ results are with the basis $N_{\max }=8$ and $K_{\max }=10$.

other hand, the magnetic form factor of the neutron shows larger differences from the data. We also presented the electromagnetic radii for the nucleon.

\section{Acknowledgments}

We thank D. Chakrabarti for helpful discussions. CM is supported by the National Natural Science Foundation of China (NSFC) under the Grant No. 11850410436. This work of XZ is supported by new faculty startup funding by the Institute of Modern Physics, Chinese Academy of Sciences under the Grant No. Y632030YRC. HL is supported by the U.S. Department of Energy under Award No. DE-FG02-93ER-40762. This work of JPV is supported by the Department of Energy under Grants Nos. DE-FG02-87ER40371, DE-SC0018223 (SciDAC4/NUCLEI), and DE-SC0015376 (DOE Topical Collaboration in Nuclear Theory for Double-Beta Decay and Fundamental Symmetries).

\section{References}

[1] J. P. Vary et al., Phys. Rev. C 81, 035205 (2010).

[2] P. Wiecki, Y. Li, X. Zhao, P. Maris and J. P. Vary, Phys. Rev. D 91, no. 10, 105009 (2015).

[3] Y. Li, P. Maris, X. Zhao and J. P. Vary, Phys. Lett. B 758, 118 (2016).

[4] X. Zhao, H. Honkanen, P. Maris, J. P. Vary and S. J. Brodsky, Phys. Lett. B 737, 65 (2014);

H. Honkanen, P. Maris, J. P. Vary and S. J. Brodsky, Phys. Rev. Lett. 106, 061603 (2011).

[5] S. J. Brodsky, H. C. Pauli and S. S. Pinsky, Phys. Rept. 301, 299 (1998).

[6] J. R. Hiller, Prog. Part. Nucl. Phys. 90, 75 (2016).

[7] Y. Li, P. Maris and J. P. Vary, Phys. Rev. D 96, no. 1, 016022 (2017).

[8] S. Tang, Y. Li, P. Maris and J. P. Vary, Phys. Rev. D 98, no. 11, 114038 (2018).

[9] S. Jia and J. P. Vary, Phys. Rev. C 99, no. 3, 035206 (2019).

[10] J. Lan, C. Mondal, S. Jia, X. Zhao and J. P. Vary, Phys. Rev. Lett. 122, no. 17, 172001 (2019).

[11] J. Lan, C. Mondal, S. Jia, X. Zhao and J. P. Vary, arXiv:1907.01509 [nucl-th].

[12] S. J. Brodsky, G. F. de Teramond, H. G. Dosch and J. Erlich, Phys. Rept. 584, 1 (2015).

[13] S. J. Brodsky, M. Diehl, D. S. Hwang, Nucl. Phys. B 596, 99 (2001).

[14] D. Chakrabarti and C. Mondal, Eur. Phys. J. C 73, 2671 (2013); Eur. Phys. J. C 75, no. 6, 261 (2015).

[15] C. Mondal, Phys. Rev. D 94, no. 7, 073001 (2016).

[16] J. Beringer et. al.(Particle data Group), Phys. Rev. D. 86, 010001 (2012). 